\title{
Closed-loop control of leading edge vorticity on a 3D wing: simulations and low-dimensional models
}

\author{
Clarence W. Rowley* $\quad$ Sunil Ahuja ${ }^{\dagger} \quad$ Kunihiko Taira ${ }^{\ddagger} \quad$ Tim Colonius $^{\S}$
}

\begin{abstract}
We study model-based feedback control of the low-Reynolds-number flow over a flat plate at large angles of attack, in both two and three dimensions. Our long-term goal is to be able to manipulate the leading-edge vortices that form on low-aspect-ratio wings at high angles of attack, and that often contribute to exceptionally large lift coefficients. In two-dimensional simulations, we present a model-based feedback controller that uses an observer to reconstruct the entire flow field from velocity measurements at three locations, and stabilizes the flow at an angle of attack for which the natural flow state is periodic shedding. In three-dimensional simulations, we use open-loop forcing to study actuator placement, and conclude that trailing-edge actuation is more effective than leading-edge actuation in influencing the forces on the plate, as well as the wake structures. Finally, we present initial results towards extending our model-based control design to the $3 \mathrm{D}$ setting, and apply a selective frequency damping method to find unstable equilibrium flow fields in 3D simulations.
\end{abstract}

\section{Introduction}

This paper presents recent results in a collaborative effort to develop model-based closed-loop flow control schemes for regulating lift produced by leading-edge vortices on wings at low Reynolds numbers. This work is originally inspired by observations that leading-edge vorticity plays an important role in lift production for insects ${ }^{1}$ and birds, ${ }^{2}$ giving rise to much higher lift coefficients than are possible in the absence of a leading edge vortex. The present collaborative effort seeks to use closed-loop flow control to regulate leading-edge vortices over both $2 \mathrm{D}$ and $3 \mathrm{D}$ wings, in order to enhance lift and provide robust flight control in the presence of gusts.

Our motivation is the control of Micro Air Vehicles (MAVs), for which gust response is particularly important, and unsteady aerodynamics plays a crucial role.

This paper focuses on recent results in simulation and modeling, for stabilizing leading-edge vortices using zero-net-mass actuators. In simulations, we model these actuators as localized body forces that act close to the surface of the wing. Previous results ${ }^{3}$ have presented low-order models of the $2 \mathrm{D}$ flow using Balanced Proper Orthogonal Decomposition (BPOD), ${ }^{4}$ a model reduction technique that approximates balanced truncation, a method popular in the control theory community, ${ }^{5}$ and has been shown to significantly outperform models determined by the more conventional Proper Orthogonal Decomposition. ${ }^{6}$ These models were used to develop feedback control laws that were able to stabilize an unstable steady-state of the Navier-Stokes equations, completely eliminating the vortex shedding, but assuming full knowledge of the flow state at all times. $^{7}$

The contributions of the present paper are as follows. We begin by extending earlier $2 \mathrm{D}$ results, ${ }^{7}$ to the case in which full flow information is not available. We use a Kalman filter to reconstruct the full flow field from three velocity sensors, and use this reconstructed flow in the feedback laws obtained earlier. The resulting output-based feedback laws are still able to stabilize the vortex shedding, although performance is not as good in the full-state-feedback case.

Our main focus is on 3D flows, since wing sweep and spanwise transport of vorticity have been shown to be critical aspects of the dynamics of leading-edge vortices. ${ }^{8}$ In Section III, we discuss open-loop control in

\footnotetext{
*Associate Professor, Princeton University, Mechanical and Aerospace Engineering, Senior Member AIAA

${ }^{\dagger}$ Graduate Student, Princeton University, Mechanical and Aerospace Engineering, Student Member AIAA

¥Postdoctoral Scholar, California Institute of Technology, Mechanical Engineering, Member AIAA

$\S$ Professor, California Institute of Technology, Mechanical Engineering, Senior Member AIAA
} 
3D simulations, comparing the effects of different actuator locations on the forces and the wake. Finally, in Section [V] we present some initial results in applying our model-based control tools to 3D flows, including an alternative method for computing unstable steady states via selective frequency damping, which appears to have a larger region of attraction than a Newton-GMRES method we have used in 2D simulations. ${ }^{3}$

\section{Observer-based feedback control in 2D}

Here, we consider the two-dimensional flow past a flat plate at a large angle of attack $\left(\alpha=35^{\circ}\right)$, at a low Reynolds number $(\mathrm{Re}=100)$ for which the natural flow state is periodic vortex shedding. Our goal is to stabilize an unstable steady state, thereby eliminating the vortex shedding, using a single actuator, here treated as a localized body force close to the leading edge of the flat plate.

We solve the Navier-Stokes equations directly (resolving all scales), using an immersed boundary fractionalstep scheme, using a multiple-grid formulation, ${ }^{9}$ on a $200 \times 200$ grid, with the smallest computational domain given by $[-1.5,2.5] \times[-2,2]$, where lengths are nondimensionalized by the chord of the flat plate, and the quarter-chord of the plate is located at the origin. We use 5 domains overall in the multiple-grid scheme, resulting in an effective computational domain $2^{4}$ times the dimensions of the smallest grid.

In our previous work, we have obtained reduced-order models using the method of Balanced Proper Orthogonal Decomposition. ${ }^{4}$ In its basic form, this method applies only to stable fixed points (e.g., steady solutions of Navier-Stokes that return to their original state when perturbed). A technique for obtaining models near unstable fixed points (e.g., steady solutions of Navier-Stokes that are not seen in nature, since they depart from the original state when they are perturbed) was presented in Ref. 7. We begin by reviewing some of these earlier results, which used full-state feedback to stabilize the unstable steady state, for comparison with our new results here, in which only limited sensor information is available. For more details about how the reduced-order models were constructed, see Ref. 7.
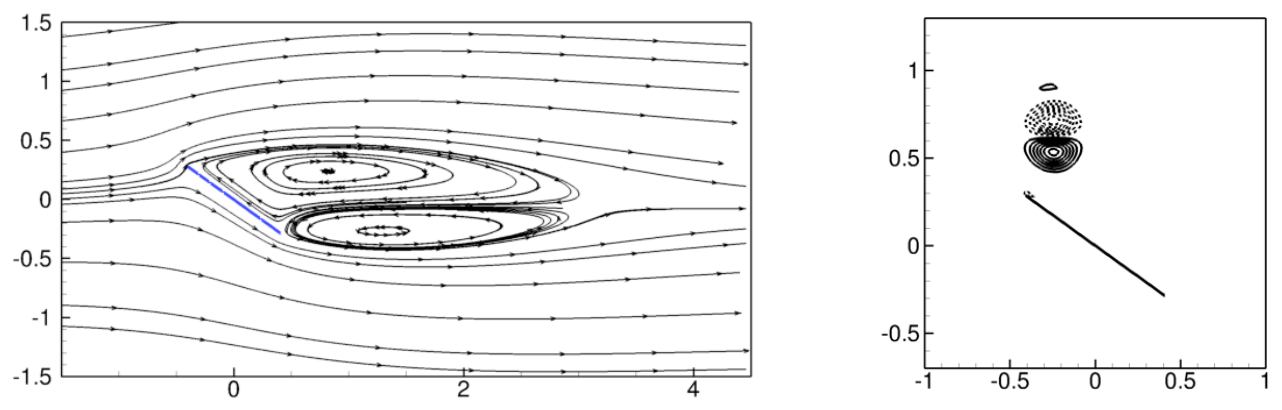

Figure 1. Streamlines of an unstable equilibrium of 2D Navier-Stokes equations, for a flat plate at an angle of attack $\alpha=35^{\circ}$ (left). Also plotted are vorticity contours of the flow field obtained on an impulsive input to the actuator (right).

\section{A. Full-state feedback control}

EQUILIBRIA The first step is to find an equilibrium solution about which to linearize. This is not straightforward for unstable equilibria, since the flow naturally wants to depart from these (for instance, producing vortex shedding). We find these unstable equilibria using a Newton-GMRES technique, ${ }^{10}$ with a timestepperbased approach ${ }^{11}$ wrapped around the Navier-Stokes solver described above. For the details of this method applied to the present flow, see Ahuja et al. ${ }^{3}$ One of these equilibrium solutions is shown in Figure 1 . If the flow is initialized exactly at this state, it remains there for all time; however, if it is perturbed only slightly, the flow starts to oscillate, and transitions to vortex shedding.

Linearized MOdels Next, we linearize about this steady state, and form low-dimensional models using Balanced POD. Our model-reduction procedure requires knowledge of the unstable eigenvectors of this 

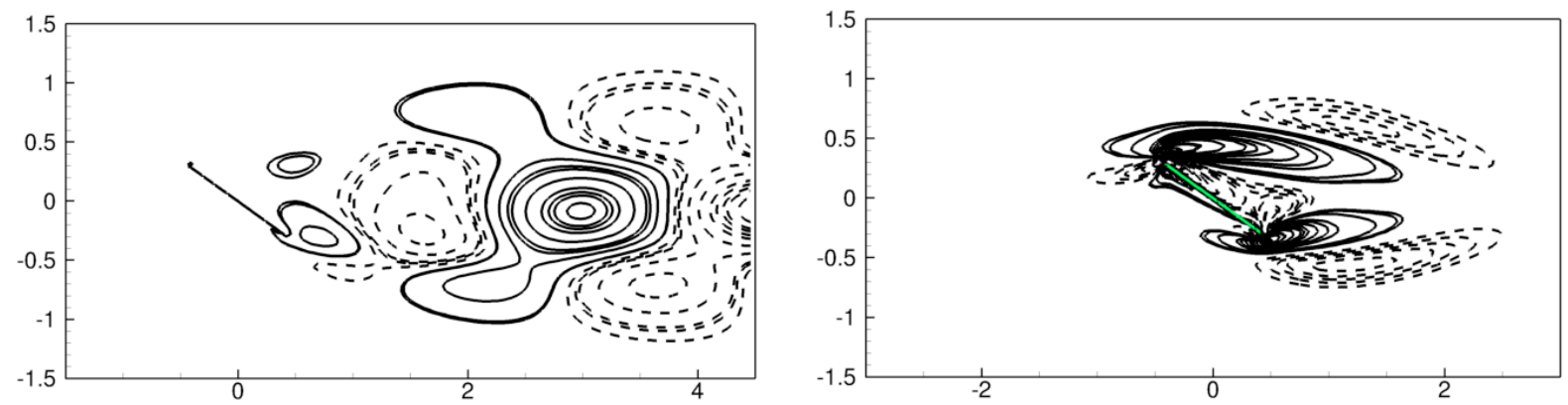

Figure 2. Real part of basis vectors of the unstable eigenspace of the linearized (left) and the adjoint (right) equations, for $\alpha=35^{\circ}$. Vorticity contours are plotted (negative contours are dashed).

linearized system. We compute eigenvectors of both the linearized and adjoint systems using ARPACK, ${ }^{12}$ and these are plotted in Figure2, The direct eigenvectors show the flow structures that emerge if the flow is perturbed from its steady state, and the adjoint eigenvectors are needed in order to project out the unstable component of the dynamics, as described below.

Impulse response simulations were then run with the system constrained to the stable subspace. This constraint is enforced by projecting the flow onto the stable subspace at each iteration, using the adjoint eigenvectors shown in Figure 2. A 6-mode BPOD model for the evolution on the stable subspace is then formed. This 6-mode model is then combined with the dynamics in the 2 unstable directions, to form a 8-mode model, from which an optimal controller (Linear Quadratic Regulator) is designed.
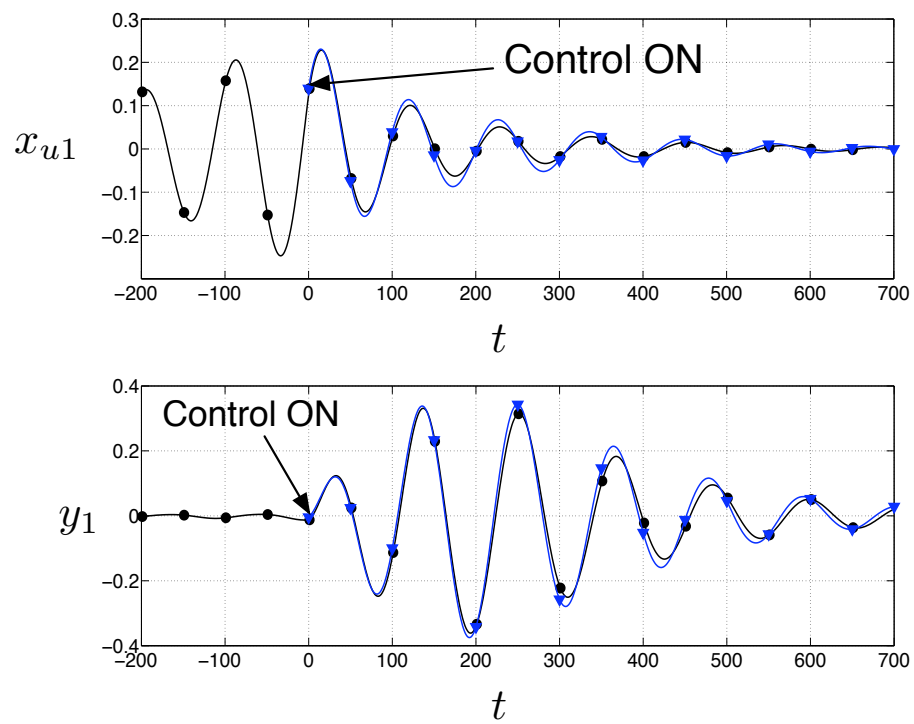

Figure 3. Time coefficients of the first unstable eigenvector (top) and first balancing mode (bottom) for a flat plate at $\alpha=25^{\circ}$, showing comparison with the full (nonlinear) DNS $(\bullet,-$,$) ) and an 8-mode model (\nabla,-,-$ When the actuator is turned on at $t=0$, the equilibrium is stabilized, and the model closely matches the full simulation.

Figure 3 shows the results of this controller implemented on the full simulation, as well as the behavior predicted by the 8-mode model. The controller is able to stabilize the unstable operating condition (the coefficients eventually decay to zero), and furthermore, the behavior closely matches that predicted by the model. For more details about this control design, once again see Ref. 7 . 


\section{B. Observer based feedback control}

The model-based controller described above is effective at stabilizing the vortex shedding. However, it requires full knowledge of the flow information at each time. Here, we develop an observer to reconstruct the flow information from a limited number of sensors. We use velocity sensors to measure both the $u$ and $v$ components in the near-wake of the plate; the sensor locations are marked by crosses in Figure 5.

Output PRojection A key step in the model reduction procedure using Balanced POD is output projection, ${ }^{4}$ useful for systems with a large number of outputs. Here, we are interested in reconstructing the entire flow field, so the output of the system is the entire velocity field. If the linearized system is written

$$
\begin{aligned}
x_{j+1} & =A x_{j}+B u_{j} \\
y_{j} & =C x_{j}
\end{aligned}
$$

then the rank of the $C$ matrix determines how many adjoint simulations are needed to construct the reducedorder model, so if this rank is too large, the number of adjoint simulations required may be prohibitive. Output projection involves defining a new system in which $C \mapsto P_{r} C$ where the new output matrix $P_{r} C$ has rank $r$ (typically small), such that the resulting transfer function is optimally close, in the $L_{2}$ sense, to the original transfer function. In this optimally close system, it turns out that the projected output is the entire velocity field projected onto the most energetic POD modes of the impulse response. ${ }^{4}$

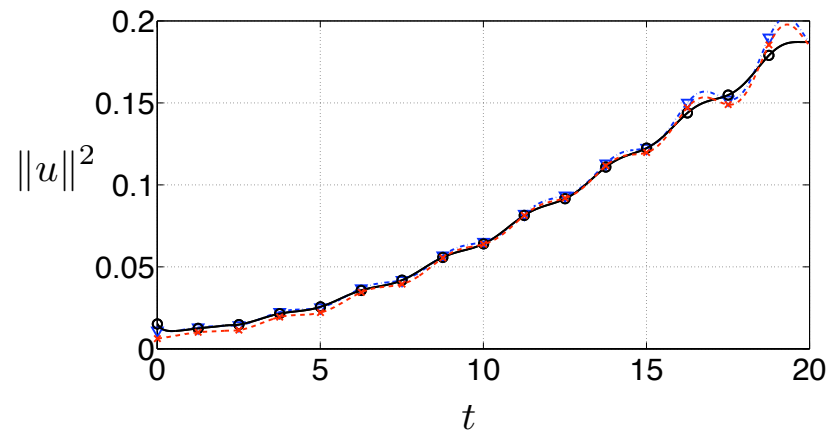

Figure 4. Evolution of the energy content, on an impulsive start, of the velocity field projected onto the stable subspace $(\circ,-)$. Also shown is the energy content of the same field projected onto the leading $4(\times,----)$ and $20(\nabla,---)$ POD modes.

The models presented in the previous section for full-state feedback were those of a system whose output was the velocity field projected onto the leading 4 POD modes. However, for observer design, this representation of the output is inadequate, as the energy content of the flow at the sensor locations is very small, while the POD modes capture the energetically dominant modes. Hence, a greater number of POD modes is required to accurately represent the velocity at the sensor locations. The temporal evolution of the energy content of the flow, obtained from an impulse response of the system restricted to evolve on the stable subspace, is plotted in Figure 4. Also plotted is the energy content of the same flow, but projected onto the leading 4 and 20 POD modes, both of which adequately represent the energy for the length of time shown. For longer times, not shown in the plot, the energy decays to zero as the system is restricted to evolve on the stable subspace. The velocity field at the sensor locations, reconstructed by 4 and 20 POD modes, are plotted in Figure 5. from which we can infer that 20 POD modes are sufficient to accurately represent the velocities at the sensor locations. Thus, we derive models using a 20-mode output projection, that is, of a system whose output is a projection onto 20 POD modes.

OBSERVER DESIGN The models obtained using the modified output are then used to design dynamic observers based on the velocity measurements at the three sensor locations. A 22-mode reduced order model, with 2 and 20 modes describing the dynamics on the unstable and stable subspaces respectively, is used to design a Kalman estimator for producing an optimal estimate of the velocity field. This estimate is then used 


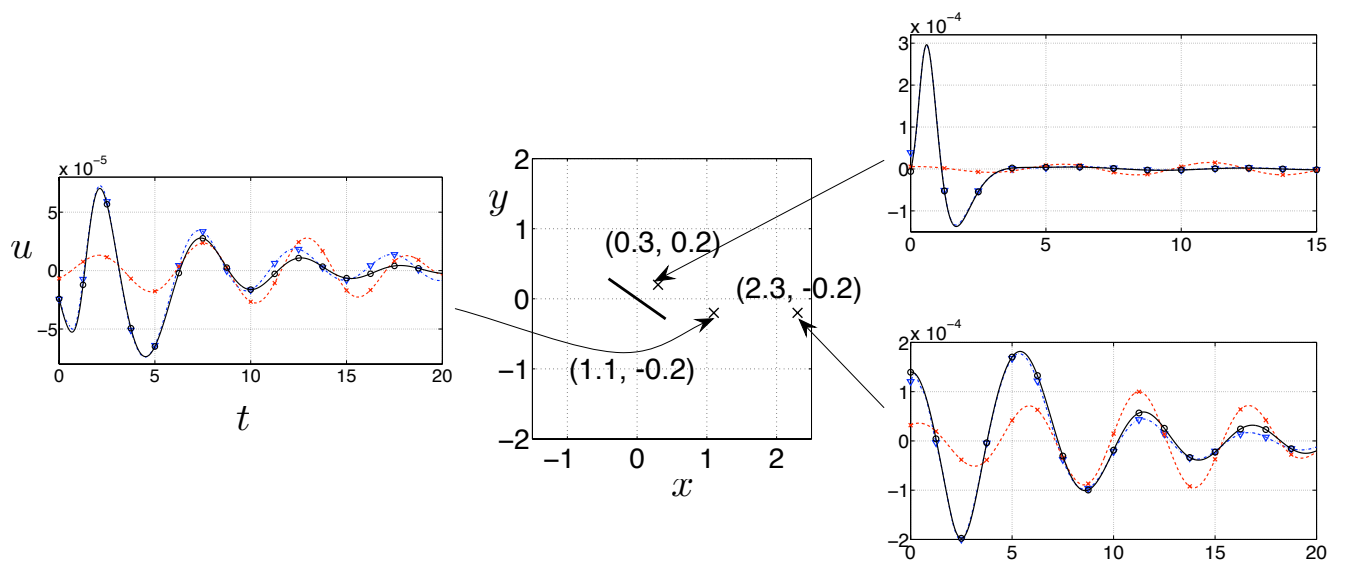

Figure 5. Velocity sensor locations (marked by crosses) used for observer design. Also shown are the timetraces of the $u$-velocity, projected onto the stable subspace, at the sensors $(0,-)$ and its reconstruction using $4(\times,----)$ and $20(\nabla,---)$ POD modes.

along with reduced order model controller to determine the control input. The results of this observer-based controller (or compensator) are shown in Figure 6. The compensator again stabilizes the unstable operating point, and furthermore, the observer reconstructs the reduced order model states accurately. Initially, the observer has no information about the states (the initial condition is zero), but it quickly converges to and follows the actual states. There is a key difference from the full-state feedback control, that the compensator does not exactly stabilize the unstable operating point but converges to its small neighborhood. The reason is that the observer design is based on the velocities at the sensor locations projected onto the leading 20 POD modes, rather than the exact velocities at these locations. These small errors enter into the observer's dynamics in the same way that sensor noise enters in, resulting in small errors in the state estimate.
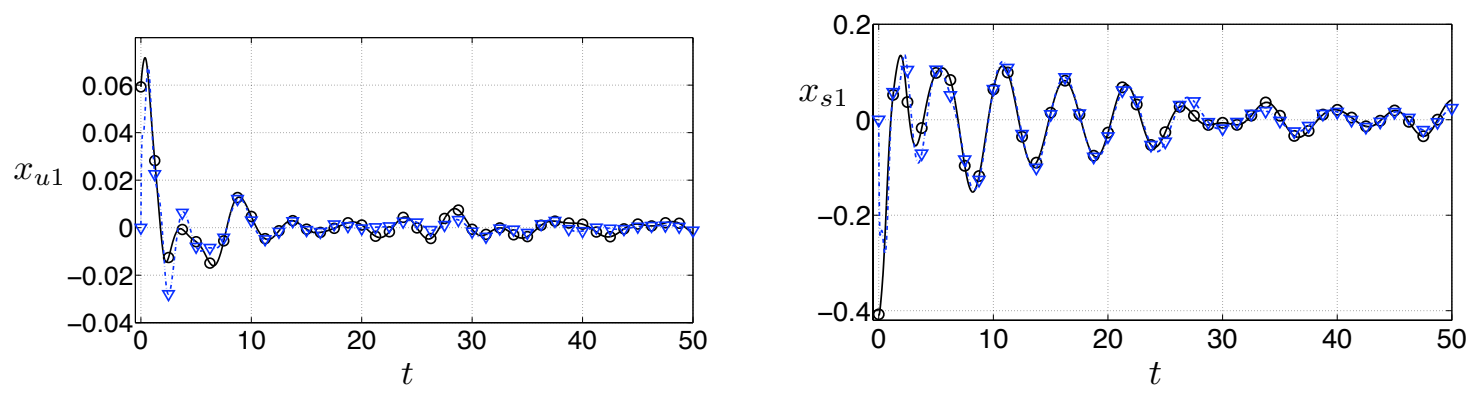

Figure 6. Observer-based stabilization of the steady state: projection of full DNS (o, - $)$, and observer prediction $(\nabla,---)$. Observer design based on a 22-mode model (2 unstable and 20 stable states, gains obtained using LQG. The initial condition is the flow field obtained from an impulsive input to the actuator, and control is turned on at $t=0$. Plots are of the velocity field projected onto an unstable eigen-direction $\left(x_{u 1}\right)$ and a projection onto the first balancing mode of the stable subspace $\left(x_{s 1}\right)$. The initial condition for the observer is zero.

\section{Simulations and open-loop control in 3D}

Our main interest is in flows around low-aspect ratio wings, for which three-dimensionality becomes important. Finite-wing effects are especially important for the vortex-shedding phenomena that we wish to stabilize, since in three dimensions, spanwise transport of vorticity is possible, and this spanwise transport influences the accumulation and shedding of vorticity, especially at the leading edge. In this section, we investigate open-loop control of these vortex shedding phenomena. 


\section{A. Three-Dimensional Simulation Setup}

A low-aspect-ratio rectangular wing is instantaneously materialized at $t=0^{+}$with its center at the origin in an initially uniform flow to replicate an impulsive translation. A typical computational domain is $[-4,6.1] \times$ $[-5,5] \times[-6,6]$ in the $x, y$, and $z$-directions (streamwise, vertical, and spanwise-directions, respectively) with a grid size of $150 \times 66 \times 156$. Grid stretching is applied to the spatial domain away from the wing. The velocity boundary condition of uniform flow is specified at the computational boundary except for the outlet where a convective boundary condition, $\partial \mathbf{u} / \partial t+U_{\infty} \partial \mathbf{u} / \partial x=0$, is applied. Validation of the computational setup is offered in Refs. 13 and 14 showing agreement between the current simulation and a tow-tank experiment for flows around an impulsively started plate of $A R=2$ at $R e=100$.

The forces on the rectangular wing of span $b$ and chord $c$ are reported in terms of the lift and drag coefficients defined by:

$$
C_{L}=\frac{F_{y}}{\frac{1}{2} \rho U_{\infty}^{2} b c} \quad \text { and } \quad C_{D}=\frac{F_{x}}{\frac{1}{2} \rho U_{\infty}^{2} b c} .
$$

The wing is modeled to be infinitely thin in the simulations with a regularized body force dependent on the grid resolution. The regularization has not been observed to influence the flow field and the body force in any significant manner. The side force generated at high angles of attack in asymmetric flow (about the midspan) is found to be at least an order of magnitude smaller than the above two forces and is not focused upon in the current study. For discussions on the side forces, we refer readers to Ref. 13.

\section{B. Uncontrolled Flow}

We consider the three-dimensional separated flow around a rectangular flat-plate wing at a Reynolds number of 300. This Reynolds number is selected as the flows around the plates are found to be unstable over a wide range of conditions while staying laminar. The wing is impulsively translated at $t=0^{+}$, in an initially quiescent flow, with a fixed angle of attack to investigate the initial transient and long-time behavior of the wake vortices and the corresponding forces exerted on the plate.

To illustrate the representative behavior of the flow around low-aspect-ratio wings, we select $A R=2$ in this section. Snapshots of the wake behind the rectangular plate of $A R=2$ are shown in Figure 7 for an angle of attack of $\alpha=30^{\circ}$. The wake vortices are visualized with two different iso-surfaces. The vorticity iso-surface is shown in light gray to capture the vortex sheets and the $Q$-criterion (or the $Q$-value), the second invariant of the velocity gradient tensor, is used to highlight the vortex cores depicted by the dark gray surfaces. Positive $Q$-values give prominence to regions of high swirl in comparison to shear to represent coherent vortices. ${ }^{15}$

\begin{tabular}{cccc}
\hline$U_{\infty} t / c=1.5$ & $U_{\infty} t / c=5$ & $U_{\infty} t / c=10$ & $U_{\infty} t / c=40$ \\
\hline & & &
\end{tabular}

Figure 7. Top-port snapshots of the wake vortices behind rectangular plates of $A R=2$ at $\alpha=30^{\circ}$. Shown are the isosurfaces of $\|\omega\|_{2}=2$ and $Q=1$.

Following the impulsive start, the vortex sheets generated by the initial acceleration and the edges of the plate roll up and form the leading-edge, trailing-edge, and tip vortices (see Figure 7 at $U_{\infty} t / c=1.5$ ). The low pressure core of the leading-edge vortex allows the plate to experience a large increase in lift. The downward induced velocity from the tip vortices presses the leading-edge vortex onto the plate until about $t \approx 8$. Around this time the leading-edge vortex detaches, interacting with the tip vortices. The columnar structure of the tip vortices is then lost and the downward induced velocity applied on the leading-edge vortices is decreased. This process causes the consequent formation of leading-edge vortices to take place farther away from the top surface resulting in reduction of lift at large time. 
The corresponding lift and drag forces exerted on the wing of $A R=2$ are shown for $\alpha=10^{\circ}, 20^{\circ}$, and $30^{\circ}$ in Figure 8. Following the impulsive start, the lift increases with the accumulation of spanwise vorticity above the top surface of the wing. The drag at $t=0^{+}$is infinite due to the impulsive acceleration. As the leading-edge vortices detach or diffuse away from the airfoils, the lift and drag are significantly decreased. Later in time, the initial columnar structure of the tip vortices are lost reducing the induced velocity upon the leading-edge vortices to stay immediately behind the plate. Hence the large-time lift is significantly lower than the lift from the initial transient. For low angles of attack (e.g. $\alpha=10^{\circ}$ ), the large-time forces are steady while for large angles of attack (e.g. $\alpha=20^{\circ}$ and $30^{\circ}$ ), fluctuations appear in the forces at large time due to the shedding of the leading- and trailing-edge vortices.
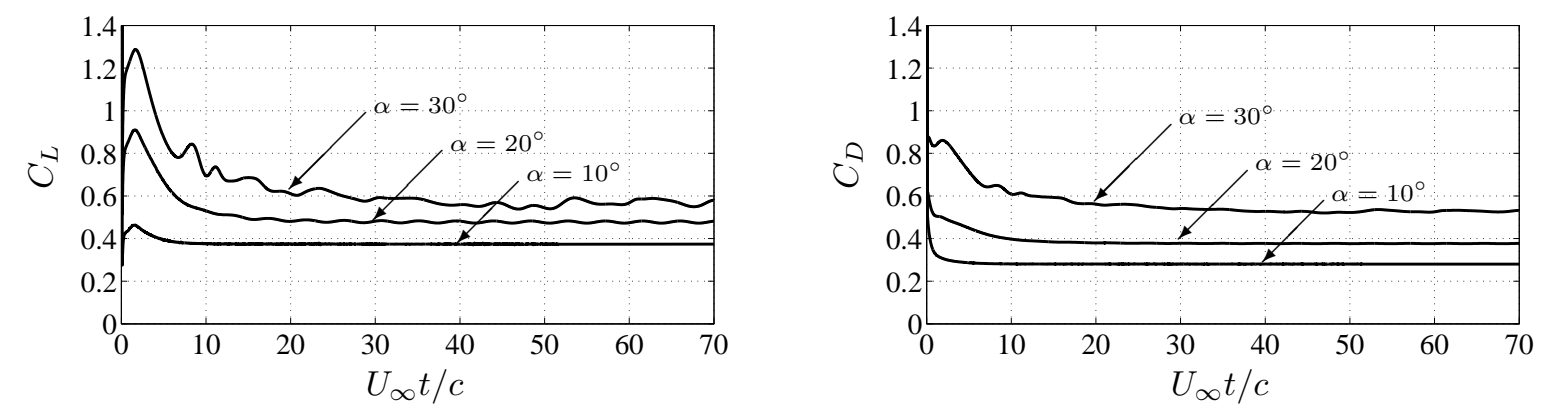

Figure 8. Lift and drag on a rectangular plate of $A R=2$ for unactuated flows of $\operatorname{Re}=300$ at $\alpha=10^{\circ}, 20^{\circ}$, and $30^{\circ}$.

\section{Controlled Flow}

As we have seen in the previous section, the lift experienced by the rectangular wing at early time following the impulsive start is significantly larger than the lift at later times. Our objective in this section is to apply steady flow control to the separated flow such that the lift at large time is increased as high as the lift attained during the unactuated transient flow. The current approach focuses on enhancing lift by changing the dynamics of wake vortices and not by reattaching the flow.

ACTUATOR MODEL In the following simulations, we introduce a body force to model steady blowing as illustrated in Figure 9. This time-invariant force is applied to the flow field as a uniform strip along the span expressed as

$$
\mathbf{f}_{\mathrm{act}}=\hat{\mathbf{f}}_{\mathrm{act}} \delta\left(x-x_{0}\right) \delta\left(y-y_{0}\right) \Gamma\left(-z+\frac{b}{2}\right) \Gamma\left(z+\frac{b}{2}\right)
$$

and is added to the right-hand side of the momentum equation. For the current model, addition of mass to the system is not taken into account. Here $\hat{\mathbf{f}}_{\text {act }}$ prescribes the strength and the direction of the actuator. The location of the actuator is specified with $\left(x_{0}, y_{0}\right)$ in the spanwise plane and $b$ denotes the span of the plate. The function $\Gamma($ ) corresponds to the Heaviside step function representing a strip in the spanwise direction. In the computations, the singular delta function, $\delta()$, is replaced by a discrete delta function, $\tilde{\delta}($ ), proposed by Roma et al. ${ }^{16}$ that regularizes the singularity across 3 cells in both the $x$ - and $y$-directions. This delta function is also used in the immersed boundary projection method to represent the immersed boundary.

The discrete delta function is selected to use the smallest possible width for the actuator, which is limited by the resolution of the simulation. Hence, the modeled slot width is set to $\sigma=\Delta x=0.04 c$, the effective width of the discrete delta function. Compared to the typical slot widths of $\sigma / c \approx 0.01$ used in flow control, ${ }^{17-19}$ the current slot width in our simulations is slightly larger. Nonetheless the present forcing function is used to explore control techniques at this low Reynolds number.

The actuator is not placed exactly on the surface of the plate to avoid the numerical interference of the boundary force used in the immersed boundary method. The controller is positioned $3 \Delta x$ from the plate on the suction side. Forcing directions of upstream, sideways (outward from the midspan to the tip), and downstream are considered. 


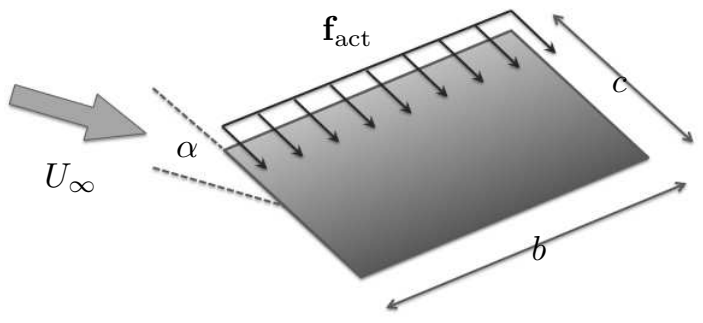

Figure 9. A flow control setup shown for an example of actuation along the leading edge in the downstream direction.

Strength of actuation The strength of the actuation is reported in the present study with the nondimensional momentum coefficient,

$$
C_{\mu} \equiv \frac{\rho U_{\mathrm{act}}^{2} b \sigma}{\frac{1}{2} \rho U_{\infty}^{2} b c}=2\left(\frac{U_{\mathrm{act}}}{U_{\infty}}\right)^{2}\left(\frac{\sigma}{c}\right)
$$

where $U_{\text {act }}$ is the actuator velocity. To characterize the actuator model, we simulate this steady blowing with a prescribed $\hat{\mathbf{f}}_{\text {act }}$ in an initially quiescent free space. Once steady state is achieved, the velocity at the center of forcing is selected as the characteristic velocity $U_{\text {act }}$. For example, $\left|\hat{\mathbf{f}}_{\text {act }}\right|=0.1$ corresponds to $U_{\text {act }} / U_{\infty}=0.356$ and $C_{\mu}=1.0 \%$.

Next, we consider the strength of actuation required to alter the flow field in a noticeable manner. In order to alter the dynamics of the wake vortices in a low-Reynolds-number flow, rather large values of $C_{\mu}$ are selected to overcome the viscous effect (and due to the large slot width limited by the grid resolution). Standard values of the momentum coefficient in past studies have ranged from $C_{\mu}=0.01 \%$ to $10 \%$ for applications of steady blowing on wings. ${ }^{17-19}$ To illustrate the change in the forces exerted upon the plate, we apply flow control around a rectangular plate of $A R=2$ at $\alpha=30^{\circ}$ and $R e=300$. This example is chosen again as the wing is at very high post-stall angle of attack generating strong interaction of the wake vortices and asymmetry about the midspan in the unactuated case. ${ }^{13}$

Actuation along the leading edge in the downstream direction with $C_{\mu}=0.10 \%, 0.51 \%$, and $1.0 \%$ are considered. With $C_{\mu}=0.10 \%$, there are no noticeable changes in the forces. As the momentum coefficient is increased to $0.51 \%$, lift starts to show increase from the unactuated case (not shown). Using $C_{\mu}=1.0 \%$, a significant increase in lift of $33.8 \%$ is observed at large time in a time-averaged sense. Hence, in what follows we consider the use of $C_{\mu}=1.0 \%$ to explore actuation locations and directions for the same example problem.

LOCATION AND DIRECTION OF ACTUATION Below we consider the application of steady blowing along the leading edge, midchord, and trailing edge in the upstream, sideways, and downstream directions (all parallel to the surface of the plate) with $C_{\mu}=1.0 \%$. Sideways actuation is directed outward from the midspan to the tips of the wing. The lift and drag histories from the controlled flows are presented in Figure 10 .

LEAding-Edge Control The top two subfigures in Figure 10 show the force histories for the cases of leading-edge actuation. Lift is increased with downstream blowing, as the separated flow structures become closer to the surface of the plate. The corresponding low-pressure vortex cores sit directly above the top surface, enhancing lift by $34 \%$ as mentioned in the previous section. The downstream blowing also repositions the wake vortices downward past the trailing edge, increasing the effective frontal area. This in turn causes the drag to increase by $16 \%$, a smaller amount compared to lift.

With flow control in the upstream direction, the leading-edge vortices are pushed into the freestream from the top surface, resulting in the loss of the low-pressure region near the surface and, accordingly, a decrease in lift. Outward forcing was applied in the hope of releasing the vorticity generated by the leading edge by inducing spanwise flow from the midspan to the tips. However, the right-angled corners on the wing separate the vortex sheets emanating from the leading edge and tip, and triggered the roll up of individual structures (i.e., the leading-edge and tip vortices). Hence the leading-edge vortex and the tip vortices remained disconnected at all times without merging or connecting, in spite of sideways blowing. 
The overall wake structure was more spread in the spanwise direction than the unactuated flow but did not have much influence on the lift or drag exerted on the plate.
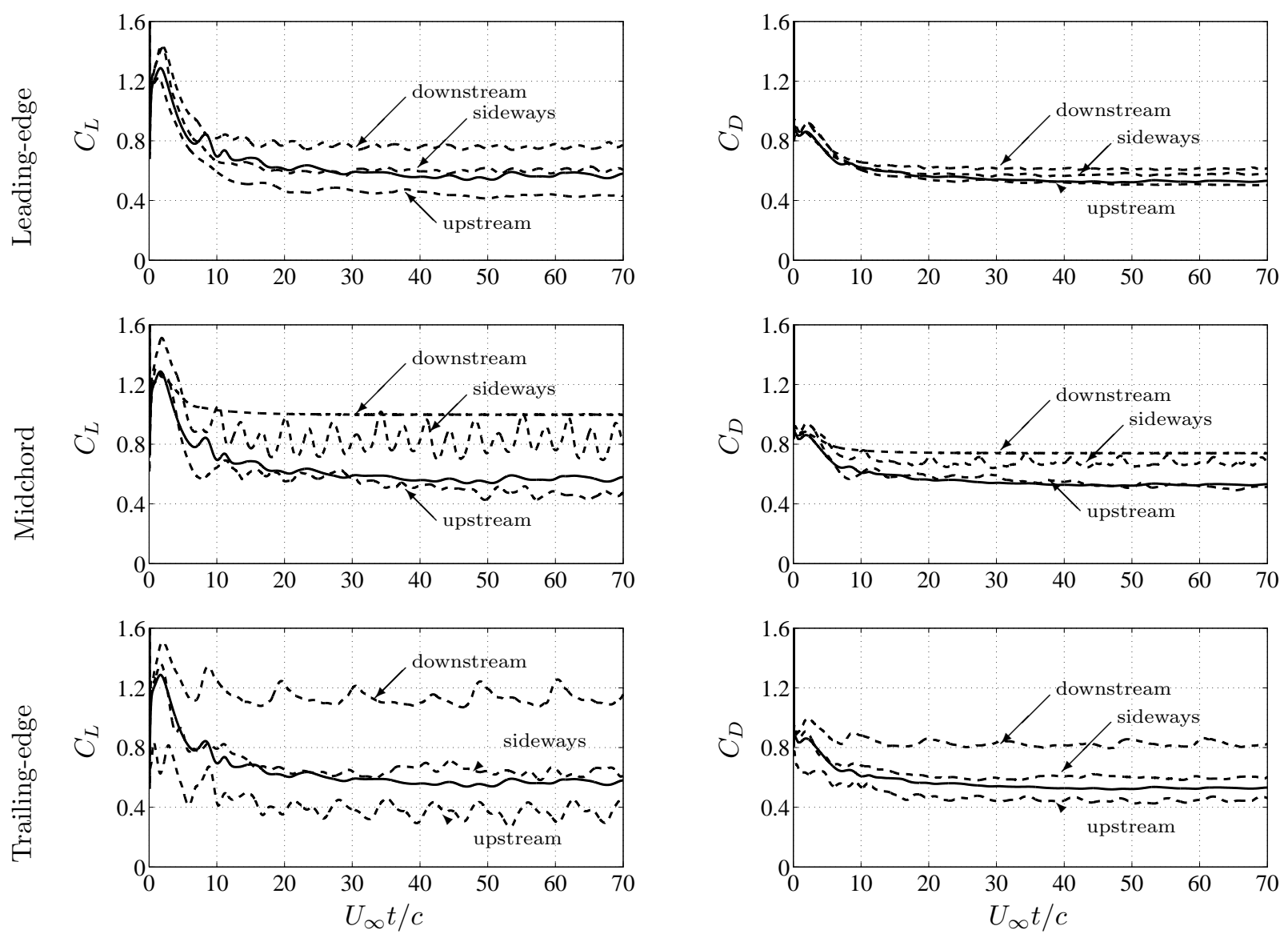

Figure 10. Lift and drag histories for cases of leading-edge, midchord, and trailing-edge actuation with $C_{\mu}=$ $1.0 \%$ applied to the separated flow around a rectangular plate of $A R=2$ at $\alpha=30^{\circ}$ and $R e=300$. Solid and dash lines correspond to unactuated and actuated cases, respectively.

Midchord Control The middle two subfigures in Figure 10 illustrate the effect of midchord actuation onto the lift and drag histories. The noticeable change from the control comes from the downward forcing where the flow achieves a steady state and a $76 \%$ increase in lift by creating an elongated wake structure (shown in the next section). The vorticity produced by the plate is stably released to the freestream from the leading-edge vortex sheet and the tip vortices at this low Reynolds number. Although this actuator setup is attractive, it would be unlikely to yield a steady flow at higher Reynolds numbers since maintaining such stable wake structure is highly dependent on viscous diffusion. We note that the drag is also affected as the wake structure is moved downward with downstream blowing.

Another change in the dynamics of the wake is exhibited by the sideways blowing along the midchord. This control arrangement repositions the tip vortices away from the plate and allows the leading- and trailingedge vortices to roll up and shed in a periodic manner. Such behavior of the flow results in a large fluctuation in the lift on the wing.

Trailing-Edge Control Among the actuation locations considered in Figure 10, the wake is found to be most sensitive to momentum injection at the trailing edge. The lift shows significant increase and decrease with the application of downstream and upstream forcing, respectively. The time-averaged lift is increased by a remarkable $100 \%$ for downstream blowing. Such noticeable changes in forces are not realized for the sideways blowing at the trailing edge. 
The reason for the strong influence of the actuation upon the forces is the direct modification of the trailing-edge vortex. Upstream and downstream blowing, respectively, encourages and discourages the interaction between the leading- and trailing-edge vortices. Below we will further examine how the downstream actuation at the trailing edge modifies the vortex dynamics in the vicinity of the wing and contributes to lift enhancement.

SumMARY OF OPEN-LOOP CONTROL RESUlTS Briefly summarizing the findings here, we have observed that steady blowing can change the dynamics of the wake vortices to increase or decrease lift and drag. From the three locations considered, the trailing edge is observed to affect the force on the wing in the most substantial manner. At all three actuator positions, steady blowing in the downstream direction enhanced lift whereas the upstream direction reduced lift. The most effective actuator for the considered example is found to be at the trailing edge in the downstream direction, achieving double the lift at large time from the change in the dynamics of the wake vortices.

\section{Wake modification with actuation}

Let us visualize the flow field around the rectangular plate of $A R=2$ at $\alpha=30^{\circ}$ and $R e=300$ for the two most effective cases of actuation from the above discussion, namely the downstream blowing at the midchord and at the trailing edge. Similar to the flow visualization employed in Figure 7, representative snapshots of the wake vortices at large time $\left(U_{\infty} t / c=70\right)$ with and without actuation are illustrated in Figure 11 with the isosurfaces of $\|\boldsymbol{\omega}\|_{2}=2$ and $Q=1$. Also presented are the time-averaged pressure fields and the streamlines along the midspan.

(a) no control
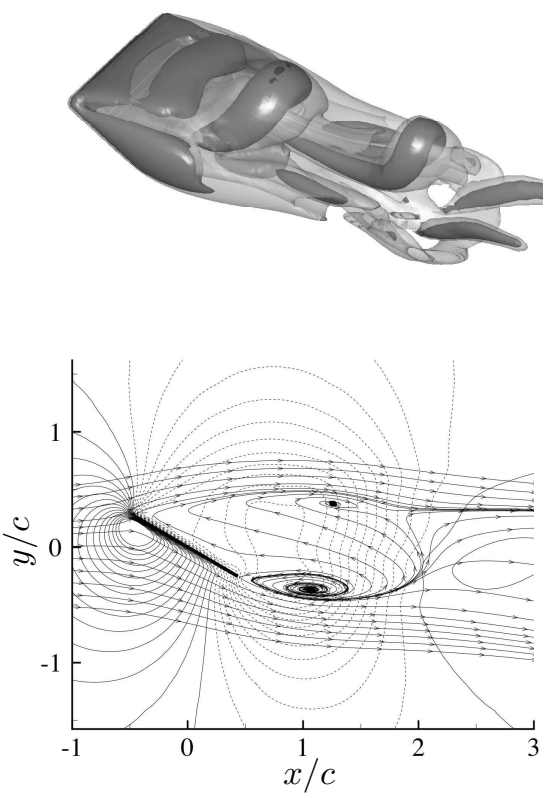

(b) midchord actuation
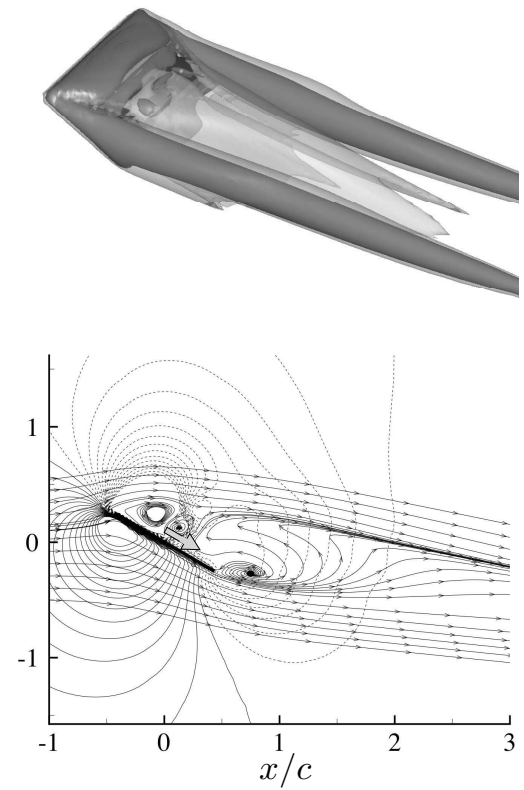

(c) trailing-edge actuation
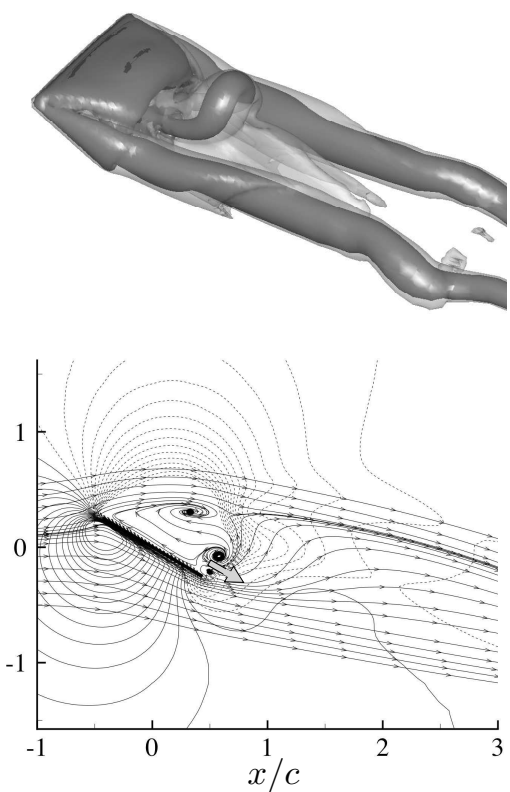

Figure 11. (Top) Snapshots of the large-time wake around a rectangular wing of $A R=2$ at $\alpha=30^{\circ}$ and $R e=300$. (Bottom) Corresponding time-averaged pressure distribution and streamlines along the midspan. Pressure contour levels are set from -0.3 to 0.3 in increments of 0.04 with the negative pressure shown by the dashed lines. Arrows indicate the location of actuation.

The unactuated flow exhibits complex interaction amongst the leading-edge, trailing-edge, and tip vortices with the dominant flow structures mostly consisting of the leading- and trailing-edge vortices. For the two controlled cases, we observe that the most dominant flow features are the long columnar tip vortices formed by engulfing the vortex sheet from the trailing edge. The downstream blowing along the midchord allows for the flow around the wing to achieve a steady state as shown with its elongated wake vortices in Figure 11(b). The 
tip vortices exhibit clear columnar structures inducing downward-induced velocity onto the wake stabilizing the leading-edge vortex sheet. The vorticity within this sheet is diffused into the free stream in a steadfast manner at this Reynolds number. In the case of downstream blowing at the trailing edge, the strengthened tip vortices apply stronger downward-induced velocity on the leading-edge vortices and let them roll up in close neighborhood of the top surface of the plate as shown in Figure 11(c). Hence, the low-pressure cores from the roll-up provide lift enhancement.

In both cases, the separation bubble with control visualized along the midspan have become smaller in a time-averaged sense compared to the unactuated case. Additionally, the streamlines are deflected further downward with blowing, directly implying that lift on the wing is increased based on the momentum balance. Note that the roll-up motion at the trailing edge from the unactuated case is now attenuated with steady blowing. The removal of such roll-up seems to be the key factor in modifying the dynamics of the wake.

Additional simulations with the same flow control setup have been performed for different wing planforms and angles of attack, all showing significant lift increase. ${ }^{20}$ In some cases, the lift has increased by more than $100 \%$. The results here have been made brief as the full three-dimensional results are reported in Refs. 13 and 20. The present emphasis has been placed on introducing the nature of three-dimensional vortex dynamics around low-aspect-ratio wings. In upcoming studies, we will explore flow control around different planform geometries, such as the semicircular wing considered in the companion studies of Williams et al. ${ }^{21}$

\section{Selective frequency-damping for computation of steady states}

In our previous work,,$^{3,7}$ we had computed a branch of steady states of $2 \mathrm{D}$ flow past a flat plate for varying angles of attack using a timestepper-based Newton-GMRES method. For 3D flows, since the number of states is much higher, this computation can be significantly more expensive. For $2 \mathrm{D}$ simulations using a coarse grid of size $50 \times 50$, the number of calls to the timestepper for computing a single steady state was around $80-100$, with 50 timesteps of integration per call. Thus, the total number of timesteps of integration is around $5 \times 10^{3}$. For a more resolved simulation using a multiple-grid method ${ }^{9}$ (grid size $=200 \times 200$ ), the number of function calls was around 200, doubling the number of integration timesteps. However, since the multiple-grid algorithm is faster, the overall computational time was actually much lower in this case. For 3D simulations, the wall-time for simulating 100 timesteps for a $40 \times 40 \times 40$ grid is approximately an hour on a single processor, which corresponds to around 2 days to compute a steady state. This calculation assumes that the number of function calls does not change for 3D. Now, since the Newton-GMRES solver needs a very good initial guess for computing the steady state, we start at a low angle of attack $\alpha \approx 10^{\circ}$ and continue the branch of steady states varying $\alpha$ in steps $\delta \alpha=0.5^{\circ}$. We are interested in computing unstable steady states at high angles of attack of $\alpha \approx 30^{\circ}$, which would require around a month of computation on a single processor. The large parameter space of $R e, \alpha$ and aspect ratio to be explored make these computational experiments more difficult. A parallel version of the code ${ }^{22}$ used in the previous section, and the $3 \mathrm{D}$ version of the multiple-grid code $^{9}$ are currently under development, and with these new tools, we expect the Newton-GMRES method to be feasible.

Here, we explore an alternate way of computing steady states, which may have a larger region of attraction than the Newton-based method, and so may not require continuing along a branch of steady states while varying a parameter. Instead, under certain conditions, we could possibly compute steady states directly at parameters of interest, such as high angles of attack. A method referred to as selective frequency damping was introduced by Åkervik et al. ${ }^{23}$ for computing steady states of the Navier-Stokes equations, and used to compute steady states of a cavity flow. We review this method briefly here.

Suppose we have a nonlinear dynamical system $q^{k+1}=f\left(q^{k}\right)$ whose steady states are given by $q_{s}=f\left(q_{s}\right)$. If the steady state $q_{s}$ is unstable, any perturbation would grow and the state will depart away from it. The frequency-damping method derives motivation from feedback control theory and involves adding a proportional term of the form $\chi\left(q^{k}-\bar{q}^{k}\right)$, where $\chi$ is a damping constant to be chosen, and $\bar{q}^{k}$ is the desired state, which is theoretically the steady state $q_{s}$. Since the goal itself is to compute the steady state, the desired state is chosen as a temporally low-pass filtered version of $q$ defined by

$$
\bar{q}^{k}=\sum_{m=-\infty}^{k} \frac{1}{\Delta} \exp \left(\frac{(m-k) \delta_{t}}{\Delta}\right) q^{m}
$$

where $\delta_{t}$ is the timestepper of the numerical solver, and $\Delta$ is the filter width, which forms another design 
parameter. Thus, the modified set of equations are:

$$
\begin{aligned}
& q^{n+1}=f\left(q^{n}\right)-\chi\left(q^{n}-\bar{q}^{n}\right), \\
& \bar{q}^{n+1}=\bar{q}^{n}+\frac{\delta_{t}}{\Delta}\left(q^{n}-\bar{q}^{n}\right),
\end{aligned}
$$

where $\chi$ and $\Delta$ are the two design parameters to be chosen. In order to select these parameters, it is helpful to investigate how the eigenvalues of the original system change upon addition of the forcing term in (7). If the linearization of the original system about the steady state $q_{s}$ is of the form $q^{k+1}=A q^{k}$, then the linearization of the modified equations 7,8 is

$$
\left(\begin{array}{l}
q^{n+1} \\
\bar{q}^{n+1}
\end{array}\right)=\left(\begin{array}{cc}
A-\chi I & \chi I \\
\left(\delta_{t} / \Delta\right) I & \left(1-\delta_{t} / \Delta\right) I
\end{array}\right)\left(\begin{array}{l}
q^{n} \\
\bar{q}^{n}
\end{array}\right) .
$$

Also, if the eigenvalue and the eigenvector of the original system are $\mu$ and $\phi$, that is $A \phi=\phi \mu$, then the eigenvectors of the modified system $(9)$ are given by $(\phi, \beta \phi)^{T}$ for a constant $\alpha$, and the corresponding eigenvalues are

$$
\begin{aligned}
\xi & =\mu+\chi \Delta(\beta-1), \\
\text { where } \quad \beta & =\frac{-F \pm \sqrt{F^{2}+4 \Delta \chi \delta_{t}}}{2 \chi \Delta}, \\
\text { and } \quad F & =\Delta(\mu-\chi)-\chi\left(\Delta-\delta_{t}\right) .
\end{aligned}
$$

Thus, the parameters $\chi$ and $\Delta$ are to be chosen such that the eigenvalues $\xi$ are inside the unit circle on the complex plane. Further, if an a priori estimate of the original eigenvalue $\mu$ is available, the optimal values of these parameters can be obtained for which the resulting values of $\xi$ have the absolute value farthest from and lower than 1 ; in other words $|\xi|-1<0$ and is minimized.

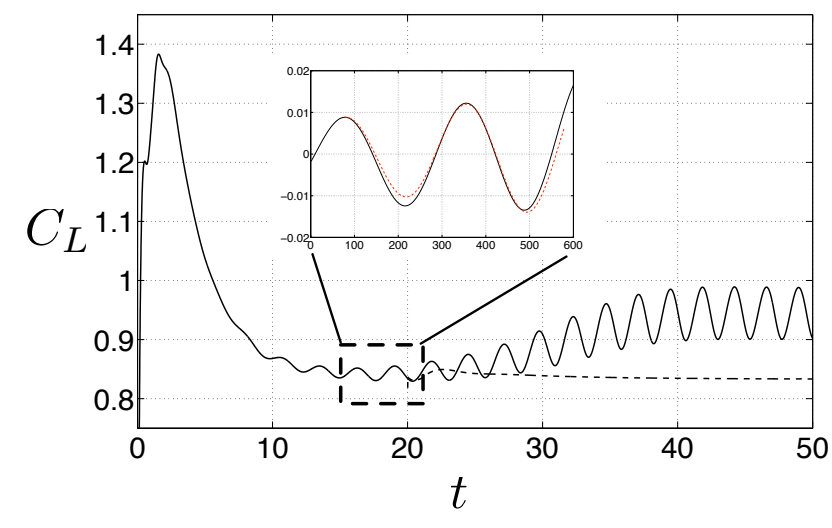

Figure 12. Time history of the lift coefficient of a rectangular plate with $A R=2$ after an impulsive start at $\alpha=14^{\circ}$ and $R e=300$. The inset shows a curve fit $(---)$ of the form $A e^{\lambda t} \cos (\omega t)$, where $\lambda+i \omega$ is an approximation of the unstable eigenvalue of the linearization about the steady state.

\section{A. Steady states of flow past a $A R=2$ flat plate.}

We present results for a $3 \mathrm{D}$ flow past a $A R=2$ flat plate at $R e=300$. The flow undergoes the first transition from steady state to periodic shedding at $\alpha \approx 13^{\circ}$. This transition takes place via a Hopf bifurcation, where a complex pair of eigenvalues crosses the imaginary axis of the complex plane from left to right (or leave the unit circle in the discrete setting). We begin by considering an operating point in the shedding region, but very close to the bifurcation, to get a good estimate of these unstable eigenvalues. We perform a simulation at $\alpha=14^{\circ}$, with an impulsive start to the plate; a time-trace of the lift coefficient is shown in 
Figure 12. At this parameter, the instability is very weak and the magnitude of the unstable eigenvalues is much smaller as compared to the rest of stable eigenvalues. As a result, the stable directions quickly decay to the unstable manifold, and the dynamics evolve on this mainfold for a short interval until the magnitude of instability becomes large enough and the nonlinear saturation becomes active in the transition to periodic shedding. The small dotted box around $t \in[15,21]$ in Figure 12 represents the time interval near the unstable manifold. Thus, a curve fit to the lift coefficient in this interval could be used to obtain an approximate estimate of the unstable eigenvalue $\mu=\lambda+i \omega$. An approximation of the lift using this estimate, $C_{L} \approx e^{\lambda t}(a \cos \omega t+b \sin \omega t)$, is plotted in red dashed line in the inset. For the discrete time-stepper with a timestep $\delta_{t}=0.01$, this eigenvalue is $\mu=1+7.22 \times 10^{-4}+0.023 i$ and the continuous counterpart is given by $\log \mu / \delta_{t}=0.099+2.3 i$.

(a)

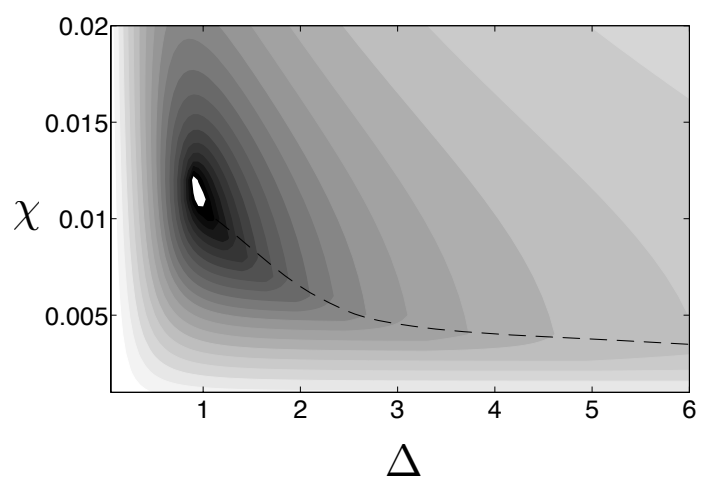

(b)

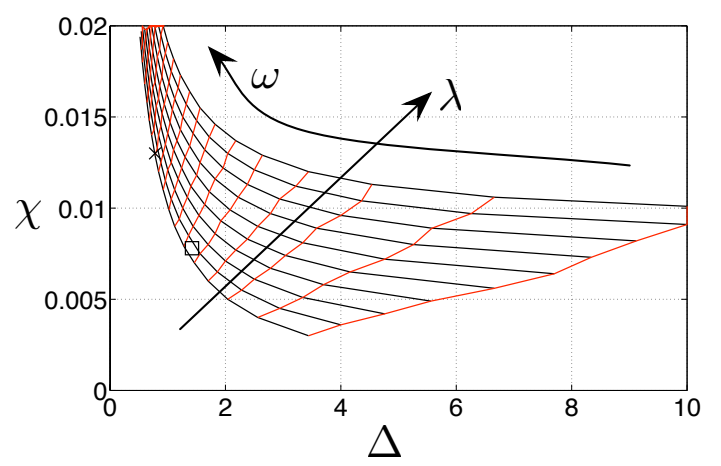

Figure 13. (a) The maximum eigenvalue of the modified system for different choices of the parameters $\chi$ and $\Delta$; plotted are contours of $|\xi|_{\max }-1$, when the eigenvalue of the original system is estimated to be $\mu=$ $1+7.22 \times 10^{-4}+0.023 i$, and the timestep $\delta_{t}=0.01$. (b) Optimal parameters for different values of the unstable eigenvalue $\mu=\lambda+i \omega$. Black and red contours represent lines of constant $\lambda$ and $\omega$ respectively. The contour levels of $\lambda$ vary from $10^{-4}$ to $10^{-2}$ in steps of $5 \times 10^{-4}$ and that of $\omega$ vary from 0.006 to 0.04 in steps of 0.002 .

Next, we use selective frequency damping in order to stabilize this steady state. For that, we solve the modified system (7, 8). These set of equations is easy to implement and is advanced in time using an explicit update. The dimension of the state is doubled due to the filtered quantity $\bar{q}$ and is a slight disadvantage of the scheme.

For selecting the parameters $\chi$ and $\Delta$, we investigate the change in the absolute value of $\xi$ for different choices of these parameters. We select the set for which $|\xi|<1$ and is the minimum. Figure 13 shows a contour plot of $|\xi|-1$ over a range of $\chi$ and $\Delta$. The contour has a minimum at $\chi=0.011$ and $\Delta=95 \delta_{t}$. Also, the contour has a cusp along the dotted line, which corresponds to the crossing of the two absolute values of $\xi$ obtained from the two choices of $\beta$ in equation 110$)$. With this set of parameters, the steady state is indeed stabilized, and the corresponding time-trace of lift coefficient is shown in Figure 14. The initial condition used is the state at $t=20$ after an impulsive start, that is the one shown in the dashed box in Figure 12. Since this state is in the proximity of the steady state, the linear analysis is valid and the control is effective in suppressing the instability. A different choice of parameters $\chi=0.01$ and $\Delta=250 \delta_{t}$ is also effective as seen in Figure 14, but the rate of convergence for these parameters is slower. The steady state thus computed is also plotted in Figure 14. where the iso-surfaces of $\|\boldsymbol{\omega}\|_{2}=2$ and $Q=1$ are plotted.

Next, we proceed to compute the steady state at $\alpha=20^{\circ}$, that is, at a parameter farther away from the bifurcation point. For this parameter, we do not have an estimate of the unstable eigenvalue as we did for $\alpha=14^{\circ}$, the reason being that the separation between the magnitudes of the unstable and stable eigenvalues isn't large. Thus, for most initial conditions, the flow quickly converges to the stable periodic vortex shedding and the dynamics do not approach the unstable manifold for any interval of time.

However, we can obtain a heuristic estimate as follows. The complex part of the eigenvalue $\omega$ can be approximated by measuring the frequency of periodic vortex shedding (for amplitudes near 1, the imaginary part can be approximated by the frequency to the first order), and the real part $\lambda$ can be linearly interpolated from its value at smaller angles of attack. An approximation can also be obtained by studying the trend 
obtained from the 2D continuation study. From these heuristic investigations, we hypothesize that the frequency $\omega$ of the instability decreases with increasing $\alpha$, while the amplitude $\lambda$ increases. For different estimates of the eigenvalue $\mu$, we obtain the optimal parameters $\chi$ and $\Delta$ that result in the most stable modified system. Different contours of constant $\lambda$ and $\omega$ in the $\chi-\Delta$ plane are plotted in Figure $13(\mathrm{~b})$. The unstable eigenvalue at $\alpha=14^{\circ}$ is marked by a cross, while the heuristic estimate of the eigenvalue at $\alpha=20^{\circ}$ is marked by a square. We use various parameters from a small neighborhood of the square and different initial conditions, but so far, the resulting simulations have not converged to the unstable steady state. One reason for this lack of convergence could be that the initial condition used is far from the linear neighborhood of the steady state and not within its domain of attraction.
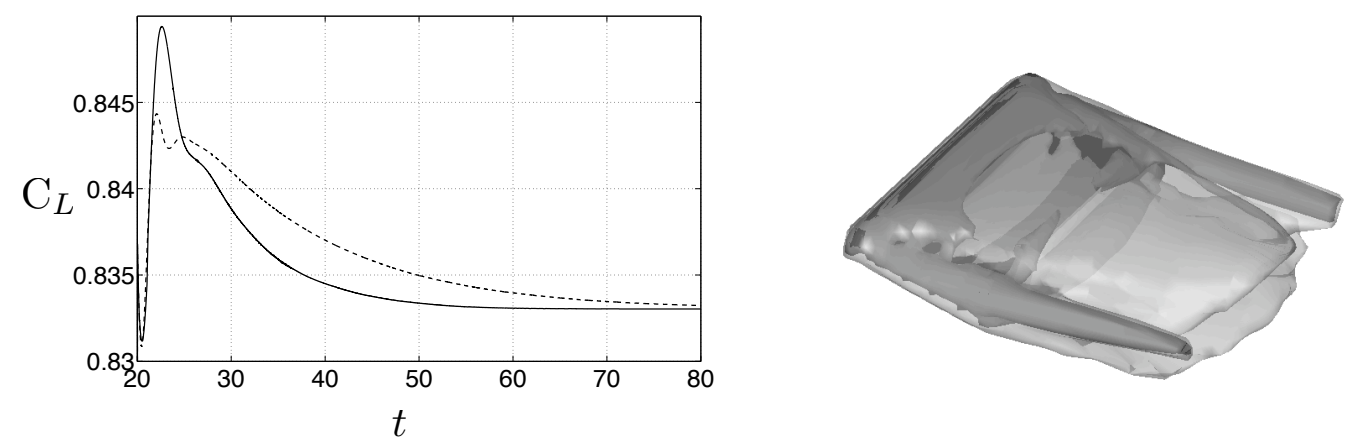

Figure 14. Lift coefficient for the frequency-damped system at $\alpha=14^{\circ}$ for two different sets of parameters: (i) $\chi=0.013, \Delta=78 \delta_{t}(-)$ (ii) $\chi=0.01, \Delta=250 \delta_{t}(\cdots \cdots)$. The parameters used for plot (i) are optimal and result in the fastest convergence to the steady state. On the right is shown the corresponding steady state; the iso-surfaces of $\|\omega\|_{2}=2$ and $Q=1$ are plotted.

\section{Conclusions}

We have discussed both open-loop and closed-loop strategies for managing shed vorticity in the vicinity of a flat plate at high angle of attack, in a low Reynolds-number flow. In 2D simulations, we have used a Kalman filter to estimate the entire flow field from three velocity sensors, and by using this observer in conjunction with a state-feedback controller, we are able to stabilize an unstable steady state, eliminating vortex shedding.

Three-dimensional simulations with open-loop forcing indicate that trailing-edge actuation is more effective than leading-edge actuation, for influencing both the overall forces on the plate, as well as the wake structures. Computing unstable steady states for the 3D flow field remains a computational challenge, but our results suggest that a selective frequency damping approach, ${ }^{23}$ when appropriately tuned, may provide a larger region of attraction than methods we have used previously, based on Newton iteration. ${ }^{3}$

In the future, we plan to develop model-based controllers for the full 3D flows, and to design observerbased feedback controllers to stabilize these unstable equilibria, thus eliminating vortex shedding in the full three-dimensional setting.

\section{Acknowledgments}

This work was supported by the AFOSR, grant FA9550-05-1-0369, with program manager Dr. Fariba Fahroo. We gratefully acknowledge Prof. Yannis Kevrekidis for helpful advice about many aspects of this work, especially the selective frequency damping.

\section{References}

${ }^{1}$ Birch, J. M. and Dickinson, M. H., "Spanwise flow and the attachment of the leading-edge vortex on insect wings," Nature, Vol. 412, Aug. 2001, pp. 729-733. 

1962.

${ }^{2}$ Videler, J. J., Stamhuis, E. J., and Povel, G. D. E., "Leading-edge vortex lifts swifts," Science, Vol. 306, 2004, pp. 1960-

${ }^{3}$ Ahuja, S., Rowley, C. W., Kevrekidis, I. G., Wei, M., Colonius, T., and Tadmor, G., "Low-dimensional models for control of leading-edge vortices: Equilibria and linearized models," AIAA Paper 2007-709, 45th AIAA Aerospace Sciences Meeting and Exhibit, Jan. 2007.

${ }^{4}$ Rowley, C. W., "Model reduction for fluids using balanced proper orthogonal decomposition," Int. J. Bifurcation Chaos, Vol. 15, No. 3, March 2005, pp. 997-1013.

${ }^{5}$ Moore, B. C., "Principal Component Analysis in Linear Systems: Controllability, Observability, and Model Reduction," IEEE Trans. Automat. Contr., Vol. 26, No. 1, Feb. 1981, pp. 17-32.

${ }^{6}$ Ilak, M. and Rowley, C. W., "Reduced-order modeling of channel flow using traveling POD and balanced POD," AIAA Paper 2006-3194, 3rd AIAA Flow Control Conference, June 2006.

${ }^{7}$ Ahuja, S. and Rowley, C. W., "Low-dimensional models for feedback stabilization of unstable steady states," AIAA Paper 2008-553, 46th AIAA Aerospace Sciences Meeting and Exhibit, Jan. 2008.

${ }^{8}$ Taira, K., Dickson, W. B., Colonius, T., Dickinson, M. H., and Rowley, C. W., "Unsteadiness in flow over a flat plate at angle-of-attack at low Reynolds numbers," AIAA Paper 2007-710, 45th AIAA Aerospace Sciences Meeting and Exhibit, Jan. 2007.

${ }^{9}$ Colonius, T. and Taira, K., "A fast immersed boundary method using a nullspace approach and multi-domain far-field boundary conditions," Comp. Meth. Appl. Mech. Eng., Vol. 197, No. 25-28, 2008, pp. 2131-46. 1995.

${ }^{10}$ Kelley, C. T., Iterative methods for linear and nonlinear equations, No. 16 in Frontiers in Applied Mathematics, SIAM,

${ }^{11}$ Barkley, D. and Tuckerman, L. S., "Stability analysis of perturbed plane Couette flow," Phys. Fluids, Vol. 11, 1999, pp. $1187-1195$.

${ }^{12}$ Lehoucq, R. B., Sorensen, D. C., and Yang, C., ARPACK Users' Guide, SIAM, 1998.

${ }^{13}$ Taira, K. and Colonius, T., "Three-dimensional separated flows around low-aspect-ratio flat plates," Journal of Fluid Mechanics, 2008, submitted.

${ }^{14}$ Taira, K., Dickson, W. B., Colonius, T., Dickinson, M. H., and Rowley, C. W., "Unsteadiness in flow over a flat plate at angle-of-attack at low Reynolds numbers," 2007, AIAA Paper 2007-710.

${ }^{15}$ Hunt, J. C. R., Wray, A. A., and Moin, P., "Eddies, stream, and convergence zones in turbulent flows," Tech. Rep. CTR-S88, Center for Turbulence Research, 1988.

${ }^{16}$ Roma, A. M., Peskin, C. S., and Berger, M. J., "An adaptive version of the immersed boundary method," Journal of Computational Physics, Vol. 153, 1999, pp. 509-534.

${ }^{17}$ Lee, C. S., Tavella, D., Woods, N. J., and Roberts, L., "Flow structure and scaling laws in lateral wing-tip blowing," AIAA Journal, Vol. 27(8), 1989, pp. 1002-1007.

${ }^{18}$ Duraisamy, K. and Baeder, J. D., "Numerical simulation of the effects of spanwise blowing on tip vortex formation," Journal of Aircraft, Vol. 43(4), 2006, pp. 996-1006.

${ }^{19}$ Holloway, A. G. L. and Richardson, S., "Development of a trailing vortex formed with spanwise tip jets," Journal of Aircraft, Vol. 44(3), 2007, pp. 845-857.

${ }^{20}$ Taira, K. and Colonius, T., "On the effect of tip vortices in low-Reynolds-number post-stall flow control," 2009, submitted, AIAA Aerospace Sciences Meeting, Orlando.

${ }^{21}$ Williams, D., Collins, J., Jankhot, C., Colonius, T., and Tadmor, G., "Control of Flow Structure on a Semi-Circular Planform Wing," AIAA Paper 2008-0597, 46th AIAA Aerospace Sciences Meeting and Exhibit, January 2008.

${ }^{22}$ Taira, K. and Colonius, T., "The Immersed Boundary Method: A Projection Approach," J. Comput. Phys., Vol. 225, No. 2, August 2007, pp. 2118-2137.

${ }^{23}$ Åkervik, E., Brandt, L., Henningson, D. S., Hopffner, J., Marxen, O., and Schlatter, P., "Steady solutions of the Navier-Stokes equations by selective frequency damping," Phys. Fluids, Vol. 18, 2006, pp. 068102. 